\title{
USO DE PLANTAS MEDICINAIS E FITOTERÁPICOS: SABERES E ATOS NA ATENÇÃO PRIMÁRIA À SAÚDE
}

\author{
USE OF MEDICINAL AND PHYTOTHERAPY PLANTS: KNOWLEDGE AND ACTS IN PRI- \\ MARY HEALTH CARE
}

\section{Ana Cristina Costa Goés ${ }^{a^{*}}$, Larissa Sonayra Leal da Silva ${ }^{b^{*}}$, Nádile Juliane Costa de Castro ${ }^{c^{*}}$}

cryszinhaK@yahoo.com.br ${ }^{\mathrm{a}}$, Larissa_sonayra@hotmail.com ${ }^{\mathrm{b}}$, nadiledecastro@hotmail.com ${ }^{\mathrm{c}}$ SESMA*, Universidade Federal do Pará ${ }^{* *}$

\section{RESUMO:}

Introdução: A atenção primária à saúde baseia-se em políticas de saúde que envolvem a cultura popular e desempenham papel importante para as tomadas de decisão dos itinerários terapêuticos, incluso neste patamar o uso de plantas medicinais e fitoterápicos. Objetivo: Este estudo tem por objetivo descrever conhecimentos e dificuldades na implantação de práticas assistências pelo uso de plantas medicinais e fitoterápicos por enfermeiros. Materiais e métodos: Pesquisa por método qualitativo desenvolvida por meio da base do modelo de estudo de caso. Foi realizado em duas unidades de saúde da família do município de Benevides, no Estado do Pará. Participaram do estudo três enfermeiros, por meio de roteiros de entrevistas referentes aos pontos elencados pelo objeto de pesquisa, distribuídos em cinco perguntas. Os dados foram coletados no período de 01 de outubro de 2015 a 20 de outubro de 2015. Resultados: O estudo revelou que os profissionais enfermeiros desconhecem as políticas direcionadas as práticas integrativas e complementares. Conclusão: Evidentemente alguns fatos foram observados como a ausência da implantação da política no município, ausência de educação permanente e continuada e pertinência do reconhecimento de implementar a assistência de acordo com a realidade local.

Palavras Chave: Cultura; Medicina preventiva; Enfermagem; Plantas medicinais; Atenção primária à saúde

\section{ABSTRACT:}

Introduction: Primary health care is based on health policies that involve popular culture and play an important role in the decision-making of therapeutic itineraries, including at this level the use of herbal and phytotherapeutic plants. Objective: This study aims to describe knowledge and difficulties in the implantation of practical assistance by the use of medicinal plants and phytotherapy by nurses. Materials and methods: Research by qualitative method developed through the basis of the case study model. It was carried out in two health units of the family of the municipality of Benevides, in the State of Pará. Three nurses participated in the study, through interview scripts referring to the points listed by the research object, distributed in five questions. Data were collected from October 1, 2015 to October 20, 2015. Results: The study revealed that nurses are not aware of policies directed to integrative and complementary practices. Conclusion: Evidently some facts were observed such as the absence of policy implementation in the municipality, absence of permanent and continuous education, and pertinence of the recognition of implementing the assistance according to the local reality.

Keyword: Culture; Preventive medicine; Nursing; Medicinal plants; Primary health care 


\section{Introdução}

O Brasil é um país com uma grande biodiversidade, e as práticas integrativas e complementares no cuidado à saúde, como o uso de fitoterápicos e plantas medicinais, são aportes que utilizam dessa particularidade. Além de ser um recurso natural, também são de baixo custo e por vezes cultivados pelos usuários dos serviços de saúde pública. ${ }^{1}$ Compreendendo sua importância e as concepções observadas dentro das políticas a serem efetivadas dentro da atenção primária à saúde é necessário apreender que os usuários tenham a orientação do uso de plantas e fitoterápicos por um profissional de saúde de modo consciente e seguro, a fim de prevenir riscos e destacar seus benefícios. ${ }^{2}$

Para melhor compreensão de tais particularidades, refere-se o entendimento da Agência Nacional de Vigilância Sanitária (ANVISA), no qual o medicamento fitoterápico é aquele obtido empregando-se exclusivamente matérias-primas ativas vegetais. Suas características apresentam eficácias e riscos, assim como pela reprodutibilidade e constância de sua qualidade. Notoriamente a sua eficácia e segurança são validadas por meio de levantamentos etnofarmacológicos, documentações tecnocientíficas ou evidências clínicas. ${ }^{3}$

Corroborando a isso, e no intuito de subsidiar seu uso, o Ministério da Saúde desenvolveu diversas ações que promoveram o estímulo a prática e utilização de plantas medicinais e fitoterápicos no Sistema Único de Saúde (SUS). Foram criadas então: a Portaria No 971 de 3 de maio de 2006 que aprova a Política Nacional de Práticas Integrativas e Complementares (PNPIC), ${ }^{4}$ o Decreto do Presidente da República No 5.813 de 22 de junho de 2006 que aprova a Política Nacional de Plantas Medicinais e Fitoterápicos, ${ }^{5}$ a Portaria No 2.960 de 9 de dezembro de 2008, que aprova o Programa Nacional de Plantas Medicinais e Fitoterápicos e cria o Comitê Nacional de Plantas Medicinais e Fitoterápicos. Todos estes dão respaldo para o uso desses na assistência à saúde e prevenção de agravos, o que deve ser efetivado a partir de seus gestores.

Com a aprovação da PNPIC, foi destacado o estabelecimento de diretrizes e responsabilidades institucionais para implantação de serviços relacionados à fitoterapia, plantas medicinais, acupuntura dentre outras práticas complementares.
Esta foi instituída com o objetivo de ampliar as opções terapêuticas aos usuários do SUS, com garantia ao acesso eficaz e com segurança, na perspectiva da integralidade de atenção à saúde, assim como de um sistema com múltiplas eficácias, incluso a participação social. ${ }^{6,7}$

Ainda, essas ações decorrentes dessas políticas, manifestadas em um Programa Nacional, são de grande importância para a melhoria do acesso da população aos medicamentos, à inclusão social e regional, ao desenvolvimento industrial e tecnológico. Ademais, permite o uso sustentável da biodiversidade brasileira, valorização, valoração, assim como da preservação do conhecimento tradicional das comunidades tradicionais e indígenas. ${ }^{8}$

Como suporte a isto, o Ministério da Saúde publicou a Portaria MS/GM no 533, de 28 de março de 2012, no qual é estabelecido o elenco de medicamentos e insumos da Relação Nacional de Medicamentos Essenciais (RENAME). Este consta de 12 medicamentos fitoterápicos contemplados. Todos eles são registrados na ANVISA e, portanto, com eficácia e segurança comprovadas. Logo, é pertinente dizer que a incorporação desses medicamentos é feita a partir da análise da sua efetividade, custo-benefício e eficácia, que são acompanhadas de regras precisas em relação a indicação e forma de uso, permitindo aos profissionais de saúde orientar a conduta de forma adequada, além de garantir a segurança dos pacientes. ${ }^{9}$

Por todos estes fatos afirma-se a pertinência da pesquisa, e, portanto, traz uma preciosa contribuição no reconhecimento, e aplicação desta terapêutica, assim como da própria política pública. Nesse ensejo, considerando estes aspectos, esta pesquisa foi realizada em uma zona rural e urbana, que apresentam diferente contextos e singularidades do usuários do serviço de saúde, inserida na realidade amazônica, com grandes evidências de práticas, saberes e atos populares.

Logo, seu objetivo é descrever conhecimentos e dificuldades na implantação de práticas assistências que provem do uso de plantas medicinais e fitoterápicos por enfermeiros. Ademais, a pesquisa visa incentivar esta prática, pelas ações dos profissionais, contribuindo pela minimização dos agravos, promovendo a saúde e revelando fatos, por meio de sua implementação em regiões urbanas e rurais. 


\section{Metodologia}

Pesquisa por método qualitativo desenvolvida por meio do modelo de estudo de caso. ${ }^{10,11}$ A escolha desse método é pertinente, pois, contribui para o conhecimento de fenômenos de indivíduos e grupos, o que permite uma visão holística quanto ao comportamento de grupos, fornecendo subsídios significativos para conduzir a pesquisa. Neste ensejo, a considerar que é estudo de um único caso, o mesmo se qualifica como do tipo de estudo de caso do tipo único holístico. ${ }^{11}$

A pesquisa foi realizada em duas unidades de saúde do município de Benevides, no Estado do Pará, que apresentam contextos diferentes em relação a sua geografia, organização espacial e cultura, ainda que se apresentem no mesmo município. Este está localizado a $33,7 \mathrm{~km}$ da capital Belém, fazendo parte da então da região metropolitana. Possui organização administrativa disposta em zonas urbana e rural, utilizada como referência para organização dos serviços de saúde. As Unidades de Saúde escolhidas foram Flores e Paraíso do Murinin, sendo a primeira localizada na zona urbana e a segunda na zona rural.

Como critério de inclusão foram considerados os enfermeiros cadastrados e atuantes no momento da pesquisa nos serviços pesquisados. Foram excluídos os em condição de férias, licença ou em regime de remanejamento e/ou condição temporária advindo de outra unidade do mesmo município e/ ou de outro. No total participaram da pesquisa os três profissionais graduados em enfermagem, atuantes nestas localidades e identificados pelos códigos Enf1, Enf2 e Enf3.

Para execução da coleta de dados foram criados roteiros de entrevistas referentes aos pontos elencados pelo objeto de pesquisa, distribuídos em três perguntas. Estas descreveram visão, aceitação, conhecimento, assim como a dificuldade para implementação de plantas medicinais e fitoterápicos nas unidades básicas de saúde. Os dados foram coletados no período de 01 de outubro de 2015 a 20 de outubro de 2015, nas unidades de saúde escolhidas no município de referência.

O percurso de análise e interpretação dos dados seguiu-se pela organização dos dados, e tomou como referência a análise de conteúdo. A pesquisa foi organizada em três fases a fim de construir um sólido resultado e uma discussão clara e objetiva. Assim sendo, em: 1) pré-análise, 2) exploração do material e 3) tratamento dos resultados, inferência e interpretação. ${ }^{12}$ Tais ponderações visaram tanto instrumentalizar, organizar e construir uma pesquisa com dados representativos.

A presente pesquisa está em consonância com as diretrizes e normas regulamentadoras de pesquisas envolvendo seres humanos, conforme solicitações éticas. A mesma foi submetida a análise ética, e aprovada pelo Conselho Nacional de Saúde sob a Resolução no 466 de 12 de dezembro de 2012 sob o parecer número 1340.078 .

\section{Resultados e discussão}

Após organização dos achados e pertinência dos elementos encontrados, direcionou-se em unidades temáticas: Conhecimento e saberes sobre Plantas Medicinais e Fitoterápicas; Do posicionamento da utilização de plantas medicinais e fitoterápicos; Conhecimento dos enfermeiros sobre a política de práticas integrativas e complementares.

\section{CONHECIMENTO E SABERES SOBRE PLANTAS MEDICINAIS E FITOTERÁPICAS}

A atuação dentro das unidades de saúde pelos profissionais de enfermagem deve sempre considerar a realidade local, a cultura e as necessidade inerentes ao modo de vida. Esta percepção deve consolidar-se pelo reconhecimento da importância do saber científico e popular sobre os temas pertinentes de acordo com a necessidade da população que receberá a assistência. Além disso, é evidente que bases legislativas e transculturais devem ser contempladas no discurso do profissional, pois a cultura é uma condição inerente ao itinerário terapêutico. Ademais, essa postura permite inferir de modo satisfatório nas ações da atenção primaria à saúde.

Por essa linha de pensamento quando questionados sobre o conhecimento sobre plantas medicinais que possuem uma frequência constante de utilização na região amazônica - pela própria realidade cultural da Amazônia, foi relatado conhecimento insuficiente por parte destes profissionais. É destacado, portanto, que muito desses apenas adquiridos pelo pequeno contato por meio de trabalhos acadêmicos e/ou 
expostos em congressos, sendo em geral superficiais para tomadas de decisão no âmbito assistencial. Em paralelo, e quando presente, foi identificado que tais conhecimentos foram repassados pelos núcleos familiares ao longo de sua formação social.

É notório por estes apontamentos que o conhecimento que o profissional de enfermagem tem sobre a utilização de plantas medicinais se mostrou em déficit, condicionado a saberes passados por relações parentais e ou eventos isolados, que é inerente a formação do ser humano. No entanto, tais evidencias demonstraram um saber científico limitado, que pode ter sido ocasionado inicialmente pela não inserção do tema pelas entidades estudantis em que foram formados os profissionais, isso em consonância a ausência de disciplinas específicas. Nesse sentido, é pertinente dizer que durante a graduação há poucas discussões acerca do tema que muitas vezes não possibilita agregar conhecimentos científicos, suficientes, pois, em geral são subtemas discutidos dentro de disciplinas bases, não específicas. ${ }^{13}$ Essas afirmativas são descritas pelas falas a seguir:

"Durante a faculdade abordaram sim, mas foi pouco, teve um trabalho, mas não foi eu que fiz" (Enf2).

"Na consulta eles sempre falam que usam, a gente passa uma medicação e eles falam: não enfermeiro eu tô tomando, mel de abelha com andiroba ou com gengibre, outro dia falaram cabacinha, eu não sabia o que era e fui perguntar pra minha avó e ela me explicou" (Enf1).

O cotidiano profissional possibilita o contato constante com a realidade da região e os saberes e práticas populares, e justamente considerando essa conjuntura que esta habilidade deve ser inserida desde sua formação acadêmica. Estes podem ser por meio de trabalhos com metodologias ativas, evitando isolamento do conhecimento por atividades ultrapassadas e realizando reflexões dialéticas. $\mathrm{Na}$ ausência de disciplina específica, a interdisciplinaridade deve ser utilizada como aporte metodológico, além da experiência do professor e de próprio projeto político pedagógico que prevê essas premissas.
Ainda, considerando os aspectos regionais, é evidente que as escolhas e ações dos usuários constantemente revelam o conhecimento e uso da medicina popular inserida em seu itinerário terapêutico. Ficou claro que não somente o usuário está envolvido nesse processo, mas também o profissional de saúde, pois, muitos possuem vivências particulares dessa realidade, e por vezes reconhecem entre seus familiares esses saberes. Nestes casos é necessário diálogo entre o saber científico e popular, de modo a transformar as práticas profissionais. Isso deve ser conduzido por meio do reconhecimento do saber popular como saber legítimo. ${ }^{14,15}$ No mais, o enfermeiro deve sempre ouvir e permitir que o usuário faça sua escolha por meio da escuta sensível, a partir das possibilidades oferecidas pelo seu saber científico e conhecimento popular próprio, promovendo ao final o poder de escolha e decisão pelo usuário.

Baseado nisto, a fala anterior revela o saber popular pelos enfermeiros por meio do uso de ervas medicinais. Neste ensejo, a erva citada é luffa operculata conhecida como buchinha do norte ou cabacinha, que é uma das plantas medicinais mais utilizada de modo terapêutico pela população, ${ }^{16} \mathrm{e}$ com efeitos relativos ao controle de agravos nasais e como abortivo natural. Entretanto, ainda pela certificação de seu uso, a maioria dos otorrinolaringologistas conhecem seus efeitos e com frequência recebem pacientes que utilizam a planta. Em geral, estes apresentam reações adversas como epistaxe, irritação nasal, alterações do olfato. Essas evidências de conhecimento popular, não devem ser desconsideradas pelos profissionais, e em verdade, devem ser registradas a fim de identificar possíveis intoxicações e eventos adversos, e estas falas demostram o conhecimento de suas efetividades.

No mais, este fato demonstra a importância de conhecer as propriedades das ervas e demais plantas de uso da população atendida nos serviços de saúde, pois essa postura implementa o uso consciente dessas. Assim sendo, é de extrema importância o reconhecimento dos efeitos adversos das plantas, para que haja orientação sobre suas interações e possíveis resultados. Sumariamente, para atender essas necessidades, as atribuições de enfermagem podem ser implementadas pela educação permanente em saúde, pois, desse modo poderá executar as orientações necessárias, as- 
sim como da conexão ao usuário pela educação em saúde. Essa conjuntura deve ocorrer de modo acessível, por meio de uma linguagem clara onde não ocorra uma confusão do entendimento entre o que deveria ser feito e o que realmente está sendo feito ao usar tais ervas.

Neste seguimento também revela o reconhecimento da enfermagem transcultural proposta por Madeleine Leininger, da legislação do SUS e do processo de humanização na assistência direta. Afirma-se isto, pois, ao considerar este cenário faz-se uma assistência orientada ao cuidado cultural. Nestes modos, se chega a real objetivo das PICS no SUS, pela implantação das políticas na prática e das considerações das realidades regionais.

Ampliando a discussão, ao conhecer as propriedades das plantas utilizadas, o profissional de enfermagem por meio de suas orientações direcionadas possibilita informações à população, direcionando-os a utilizar produtos sem nenhuma indicação e orientação. Nesses modos, ao implementar estes saberes, tanto os enfermeiros como outros profissionais, tendem a aproximar o conhecimento científico e popular, respeitando a realidade na qual se está inserido as tomadas de decisão terapêutica. Favoravelmente, com base no reconhecimento das plantas e fitoterápicos que já são utilizados, estes profissionais poderão realizar uma orientação eficaz, garantindo a segurança de seu uso e aplicando os processos previstos na legislação do SUS quanto a equidade.

Mas, para que essa prática seja assegurada, a educação/formação dos profissionais em plantas medicinais e fitoterapia de acordo com a PNPIC deve ser realizada, e isto poderá ocorrer por meio de curso básico para toda a equipe. Estas visam aspectos gerais, de manipulação e a sensibilização dos profissionais. Além disso, é necessário incluir disciplinas sobre fitoterapia e outras práticas complementares nos cursos de graduação e especialização, a fim de ampliar a discussão desde a formação profissional. Não ausência de tais debates, temas referentes a fitoterapia, podem nem ser de conhecimento dos profissionais como se revela na fala a seguir:

"Me explica o que é isso?" (Enf1). "O que é fitoterapia?" (Enf2).
Dúvidas como as registradas na pesquisa só evidenciam a falência de discussões mais efetivas nessa temática. Porém, situações que mostram ausência de conhecimento tem como aporte a possibilidade de atividades de educação permanente pelos serviços, assim como da educação continuada pelo profissional, haja vista que hoje há diversos mecanismos de atualização, seja por eventos científicos ou por plataformas on line. Como grande evento nesse sentido e para afirmar essa condução, no ano de 2017 foi realizada na capital Brasília o Congresso de práticas integrativas e complementares em saúde, sendo um espaço de aprendizado, discussão e trocas de saberes. Foi um espaço que inclusive oportunizou a participação de representações tradicionais como dos indígenas.

Há, portanto, de se considerar que discursos como os revelados nas falas exprimem o desconhecimento das várias possibilidades terapêuticas das práticas complementares, principalmente das plantas medicinais, e acabam deixando uma lacuna assistencial, podendo colocar a população sob risco. Sim, obviamente que o profissional deverá sempre ter seu desenvolvimento intelectual em constante atualização, pois, é uma necessidade observada em todas as etapas da carreira, mas como encontrado nesta pesquisa, não houve essa busca.

Mas sobretudo, esta conduta também facilita as rotinas do local de trabalho quando a condução das políticas em implantação, haja vista que o reconhecimento das políticas nacionais de saúde permite uma maior compreensão no processo de inserção local. ${ }^{17}$ Vale ressaltar, portanto, que a busca por novas descobertas promove o desenvolvimento profissional, e são ações que devem ser realizadas em conjunto com as iniciativas promovidas pelos gerentes dos serviços. ${ }^{18}$

\section{Do posicionamento sobre a utilização de plantas medicinais e fitoterápicos}

Ainda que lhes faltem conhecimento científicos sobre o tema proposto, foi identificado que os mesmos são favoráveis a implementação da PNPIC no sistema de saúde. Porém, entendem que a implantação das políticas e ações de modelos que envolvem terapêuticas complementares, deve 
estar acompanhada de capacitação realizada por profissionais maduros, e que implementem ações estratégicas com apoio dos gerentes de saúde. Logo, os enfermeiros pesquisados reconhecem a importância da utilização das plantas medicinais, já que entendem que é um modo de tratamento notório na realidade local, contudo requerem ser capacitados por facilitadores com experiência.

As unidades de saúde por se apresentarem como um espaço primário e um ambiente onde os fármacos naturais podem ser discutidos pelo profissional e o usuário do SUS, é um local democrático em que as políticas de saúde, inclusive das PNPIC devem ser priorizadas. ${ }^{19}$ Logo, considerando um espaço de democracia, os saberes dos usuários devem ser considerados pelo enfermeiro durante suas condutas terapêuticas, ${ }^{20}$ não somente por estar atrelado a uma política, mas por ser uma condição cultural que envolve escolhas e realidades. E ainda, não menos importante, pelo fato dos enfermeiros estarem inseridos na porta de entrada dos serviços de saúde por meio da atenção primária, este é responsável pelo primeiro contato que sensibilizará o usuário para o tema, o que deveras ser fator planejado e orientado de modo satisfatório.

A Atenção Básica a Saúde (ABS) é responsável pelo primeiro contato do usuário com o serviço público de saúde e está pautada em princípios como a descentralização, universalidade, equidade e participação social. Caracteriza-se também, por ser a principal porta de entrada e ponto estruturante do processo de comunicação entre toda rede de atenção à saúde sendo responsável pela continuidade do cuidado e construção do vínculo entre profissional de saúde e usuários (FERREIRA, 2017, p. 36).

O enfermeiro tem se destacado no serviço da atenção primária e como tal deve reconhecer os princípios que norteiam o SUS, justamente para considerá-los no cuidado à saúde dos usuários, incluindo a PNPIC. Contudo, algumas situações devem ser destacadas, pois, no caso deste estudo, os achados apontam que os enfermeiros descrevem ser favoráveis à utilização de plantas medicinais, desde que os usuários não deixem de tomar os medicamentos laboratoriais prescritos, ou seja, utilizar de formar associada, o que considera os itinerários terapêuticos, mas impõe condições. Essa condição é percebida em algumas falas, e inclusive, refere o uso das plantas medicinais atrelado a ausência de efeitos tóxicos, e por isso liberado para consumo, como comprova as falas a seguir:

"Olha eu respeito, se alguém chegar falando que vai tomar um chá de alho pra baixar a pressão, não tem o menor problema, desde que tome seu losartana" (Enf3).

"Como é natural não agride o organismo e não causa dependência total da droga em si"(Enf2).

A percepção de respeito pelo enfermeiro afirma as bases da humanização do SUS, o reconhecimento de um itinerário terapêutico e dos fazeres populares. Isso ocorre porque entende e institui por meio de uma assistência equilibrada o entendimento das necessidades do usuário, ainda que haja processos de imposições de condições de uso. Ainda assim, mesmo com esses discursos, favorece o resultado da assistência, pois, dá condições para a melhora da relação interpessoal com seus usuários, promovendo mais facilmente o diálogo. Mas, deixa-se claro aqui que não é a melhor postura, e por tal deve ser revista, permitindo que o usuário faça sua própria escolha como já afirmado.

Neste sentido, é entendido que deverá haver uma articulação entre os usuários e os serviços de saúde, sendo sua concepção integral. ${ }^{21}$ Esta visão se distancia da condição fragmentada, colocando essa conexão dentro dos aspectos socioambientais e de humanização. São condições que se organizam dentro dos princípios da universalidade, equidade e na prática da promoção e prevenção à saúde.

Vale ressaltar, que o posicionamento do enfermeiro perante as políticas de saúde previstas é condicionado ao seu conhecimento científico, suas vivências e as próprias concepções quanto ser social. A percepção do enfermeiro deve ser pautada em uma visão integral e global dentro de sua assistência à saúde, e por vezes, diferente de sua realidade pessoal. É quando as bases teóricas devem estar bem claras quanto ao cuidado cultural e ao processo saúde-doença, que por vezes não foram inseridas na formação acadêmica. Contudo, quando 
o enfermeiro não entende ou não obteve recursos teóricos para compreender essa realidade, as práticas acabam por serem direcionadas a atividades tecnicistas e atreladas ao modelo biomédico.

\section{Conhecimento dos enfermeiros sobre a política de práticas integrativas e complementares}

Apesar da PNPIC tem sido publicada em 2006 ainda há pouca divulgação nas mídias digitais por parte dos órgãos responsáveis. Isto pode até implicar em uma condição do desconhecimento da política por muitos enfermeiros, mas não o justifica. É fato que há plataformas on line e outros modos de atualização na atualidade, e onde esses profissionais devem sempre estar buscando informações. Contudo, o estudo nos revelou que isso não está ocorrendo, pois, foi constatado que os profissionais desconheciam a Política de Práticas Integrativas e Complementares, conforme descrito a seguir:

\section{"Nunca ouvi falar dela" (Enf1). "Não conheço" (Enf2). "Não conheço ela"(Enf3).}

Como mencionado anteriormente, o profissional de saúde deve primeiramente ter contato com esse tema em sua formação acadêmica. Esta condição promove além de habilidades a conscientização da importância sobre o tema e é inerente as habilidades e competências que esses devem possuir para a prática de enfermagem. Contudo, de sua ausência, é pertinente afirmar que é de obrigação do profissional atualizar-se continuamente a fim de permitir uma assistência segura, incorporada por práticas atualizadas e dentro das diretrizes do SUS.

A enfermagem, como uma profissão que busca o cuidado integral ao ser humano, percebendo a influência dessas práticas sobre a comunidade, deve incorporar as modalidades terapêuticas complementares. O exercício dessas terapias está regulamentado pelas Resoluções 197/97 e 283/03 do Conselho Federal de Enfermagem, o que possibilita ao enfermeiro utilizá-las nos diferentes contextos de atenção à saúde. ${ }^{22}$ Então, há subsídios suficientes para seu engajamento nas atividades do SUS, não havendo prerrogativas para sua ausência.

A PNPIC é uma estratégia revolucionária, pois visa promover a saúde da população utilizando as plantas medicinais, é uma peça de resgate da cultura e uma forma de aproximação dos profissionais de saúde, setores governamentais e população. Esta prática sensibiliza para a aproximação e incentiva o respeito entre as partes - cuidadores e cuidados - possibilitando aos enfermeiros que ensinem o que sabem e aprendam com a cultura dos usuários. Entretanto, devem estar dispostos a se envolver nessa nova relação terapêutica, priorizando a saúde e não a doença, portanto, as próprias PNPIC podem ajudar nesse sentido, como uma prática de prevenção dos agravos.

Observando essa realidade, destaca-se que a enfermagem é uma profissão que se caracteriza pela amplitude de suas práxis, justamente por ir além das ações nos consultórios, ambulatórios e afins, mas, que engloba práticas como das visitas domiciliares. Esta é uma atividade oportuna em saúde coletiva para realizar ações da enfermagem. ${ }^{23,24,25}$ É por meio delas que se pode observar a melhor maneira de cuidar de seus clientes, direcionando o cuidado e levando em consideração seu próprio ambiente e suas escolhas. Logo, se o usuário preferir a utilização de plantas medicinais e/ou fitoterápicos, os enfermeiros devem estar preparados para orientá-lo de acordo com sua necessidade.

Portanto, a capacitação para o desenvolvimento das práticas integrativas e complementares se torna uma necessidade não somente dos enfermeiros, pois, visa à integralidade do cuidado. A postura pela busca por especialização nessas áreas pelos profissionais, também, pode representar uma mudança social profunda em relação ao cuidado em saúde, envolvendo transformações culturais mais amplas. ${ }^{26} \mathrm{~A}$ continuidade de aprendizado deve ser priorizada, e justamente percebeu-se que os que foram pesquisados demonstram interesse na temática, porém não buscam conhecimento sobre $o$ assunto.

A falta de iniciativa, contudo, acaba por distanciar o mesmo da rotina cultural da comunidade. É importante, portanto, que os profissionais de enfermagem reconheçam as culturas e costumes da população, e para isso é necessário que esteja 
capacitado para orientar as equipes saúde da família, ${ }^{27}$ ideia esta que pode ser recebida desde a construção das habilidades profissionais. ${ }^{13}$ Há de se buscar sempre campos que possam subsidiar esses processos, e justamente a saúde coletiva vem intervir por meio de seus instrumentos nestas realidades, considerando as diversas dimensões. ${ }^{28}$ Obviamente que essa ideia deve ser partidária dos gerentes dos serviços e reconhecidas tanto por estes como pelos profissionais de enfermagem. Assim, compartilha-se da ideia da necessidade de iniciativas de educação permanente para qualificação do profissional. ${ }^{29,30}$

\section{Conclusão}

O estudo revelou que os profissionais enfermeiros desconhecem as políticas direcionadas as práticas integrativas e complementares. Evidentemente alguns fatos foram observados como a ausência da implantação da política no município, ausência de educação permanente e continuada e pertinência do reconhecimento de implementar a assistência de acordo com a realidade local. Considerando todos estes fatos, é pontual afirmar que o ministério da saúde possui inúmeros incentivos na prática de terapias complementares e $\mathrm{O} \mathrm{CO}$ FEN reconhece e habilita os enfermeiros a utilizarem deste recurso. Contudo, é necessária atuação direta dos órgãos governamentais, profissionais e do próprio enfermeiro não nesse processo.

Novos conhecimentos possibilitam que os enfermeiros ampliem seu respeito a cultura e a forma de vida da população, e os aspectos envolvidos no espaço promovedor de saúde devem ser levados em consideração, para que não se confunda respeito com omissão, pois a população tem um amplo conhecimento empírico, porém é carente de informações científicas. É necessário sempre aproximar a cultura dos clientes e afastá-los de riscos pelo conhecimento acadêmico, de modo responsável, e dentro das competências e princípios da enfermagem. Ademais, estudos nessa área devem ser conduzidos a fim de investigar outras condições que estejam atreladas ao déficit de conhecimento desta política de modo mais específico de acordo com as questões socioculturais das áreas de atuação da equipe de saúde.

\section{REFERÊNCIAS}

1. Iank MA, Moraes EF, Mezzomo TR, Oliveira VB. Conhecimento e uso de plantas medicinais por usuários de unidades básicas de saúde na região de Colombo-PR. Revista Saúde e Desenvolvimento. 2017; 11(8): 29-30.

2. Rodrigues MVN, Oliveira ADSS, de Paula Lourenço JO, Pereira B, Rodrigues RAF, Figueira GM. (2016). Aprendendo a transformar plantas medicinais em fitoterápicos seguros. Sínteses: Revista Eletrônica do SIMTEC. 2016; (6): 219 .

3. Ministério da Saúde (BR). Agência Nacional de Vigilância Sanitária. Resolução - RDC n.10, de 9 de março de 2010. Dispõe sobre a notificação de drogas vegetais junto à Agência Nacional de Vigilância Sanitária (ANVISA) e dá outras providências. Diário Oficial da União. Brasília (DF). Ministério da Saúde; 2010.

4. Ministério da Saúde (BR). Política Nacional de Plantas Medicinais e Fitoterápicos. 1. ed. Brasília (DF) série B, 2006. $60 \mathrm{p}$.

5. Brasil. Ministério da Saúde. Presidência da República, Casa Civil. Decreto 5.813 de 22 De junho de 2006. Aprova a Política Nacional de Plantas Medicinais e Fitoterápicos e dá outras providências. [internet] 2006 [acesso em 2015 Mar 28] Disponível em: http://www.planalto.gov. br/ccivil_03/_Ato2004-2006/2006/Decreto/D5813.htm

6. Rodrigues AG, Santos MG; Amaral ACF. Políticas Públicas em Plantas Medicinais e Fitoterápicos. In: BRASIL. Ministério da Saúde. Secretaria de Ciência, Tecnologia e Insumos Estratégicos. Departamento de Assistência Farmacêutica. A fitoterapia no SUS e o Programa de Pesquisa de Plantas Medicinais da Central de Medicamentos. Brasília: Ministério da Saúde, 2006. 148 p. (Série B. Textos Básicos de Saúde).

7. 7.Martins F, Bôas GV, Rocha L. Estudo da PNPIC e da PNPMF e seus reflexos no Estado do Rio de Janeiro. Revista Fitos Eletrônica. [internet] 2015 [acesso em mai 2016]; 9(4): 279-282. Disponível em: http://revistafitos.far.fiocruz.br/index.php/revista-fitos/article /view/276/pdf_81 doi: http://dx.doi.org/10.5935/2446-4775.20150024

8. Ministério da saúde (BR). Programa Nacional de Plantas medicinais e Fitoterápicos. 1. ed. Brasília: Ministério da Saúde; Série C, 2007. 136 p.

9. Ministério da saúde (BR). Caderno de atenção básica. Plantas Medicinais e Fitoterapia na Atenção Básica. 1. ed. Brasília: Ministério da Saúde; n. 31, 2012. 156 p.

10. Creswell JW. Research design: Qualitative, quantitative, and mixed methods approaches. London (UK): Sage publications; 2013 .

11. Yin RK. Estudo de caso: planejamento e métodos. Porto Alegre (RS): Bookman; 2016

12. 12.Bardin L. Análise de conteúdo. São Paulo (SP): Edições 70; 2016 
13. De Castro NJC, Cavalcante IMS; Palheta ASE, Santos DN. Inclusão de disciplinas em graduação de enfermagem sobre populações tradicionais amazônicas. Cogitare Enfermagem. [internet] 2017 [acesso em 2018 jan 11]; 22(2) e49730. doi: http://dx.doi.org/10.5380/ce.v22i2

14. Acioli S, Kebian LVA, Dias JR, Corrêa VAF, Daher DV, Martins ALX. Saberes científicos e populares na Estratégia Saúde da Família na perspectiva hermenêutica-dialética. Online braz j nurs. 2016;15(4): 644-54.

15. Badke MR, Somavilla CA, Heisler EV, De Andrade A, Budó MDLD, Garlet TMB. Saber popular: uso de plantas medicinais como forma terapêutica no cuidado à saúde. Revista de Enfermagem da UFSM. [internet] 2016 [acesso em 2017 jun 11 ]; 6(2): 225-234. doi: http://dx.doi. org/10.5902/2179769217945

16. Baldiserra MD, Copetti PM, Oliveira PSB, Sagrillo MR. Efeito genotóxico in vitro do extrato aquoso de luffa operculata sobre células mononucleares de sangue periférico. Disciplinarum Scientia| Saúde. 2016;15(1): 1-10.

17. Price S, Reichert C. The Importance of Continuing Professional Development to Career Satisfaction and Patient Care: Meeting the Needs of Novice to Mid- to Late-Career Nurses throughout Their Career Span. Adm. Sci. [internet] 2017 [acesso em 2017 mai 15]; 7(17):17. doi: https://doi.org/10.3390/admsci7020017

18. Inge A, Pool RF, Poell Marjolein GMC. Motives and activities for continuing professional development: An exploration of their relationships by integrating literature and interview data . Nurse Education Today. [internet] 2016 [acesso em 2017 mai 24]; (38): 22-28. doi: https:// doi.org/10.1016/j.nedt.2016.01.004

19. Ferreira FN. Conhecimento e usos de plantas medicinais e suas interconexões com a saúde pública do município de Arauá/SE. [Dissertação] Sergipe: Universidade Federal de Sergipe; 2017. 141p.

20. Paiva CHA, Teixeira LA. Reforma sanitária e a criação do Sistema Único de Saúde: notas sobre contextos e autores. Revista História, Ciências, Saúde - Manguinhos. [internet] 2014 [acesso em 2016 out 20 ]; 21(91):15-35. doi: http://dx.doi.org/10.1590/S0104-59702014000100002

21. Silva RCM. Relações produtoras do cuidado em práticas com plantas medicinais na estratégia saúde da família em Juazeiro /Bahia. [Tese]. Sergipe: Universidade Federal de Sergipe; 2016. $174 \mathrm{f}$.

22. Conselho Federal de Enfermagem. Resolução 197/1997. Estabelece e reconhece as Terapias Alternativas como especialidade e /ou qualificação do Profissional de Enfermagem. Parecer normativo do COFEN n ${ }^{\circ}$ 004/95. Documentos básicos de enfermagem. São Paulo; 1997 [acesso 2015 nov 20]. Disponível em: http://www.cofen.gov.br/ resoluo-cofen-1971997_4253.html
23. Egry EY, Fonseca RMGS. A família, a visita domiciliária e a enfermagem: revisitando o processo de trabalho da enfermagem em saúde coletiva. Rev Esc Enferm USP. [internet] 2000 [acesso em 2017 jan 18]; 34(3): 233-9. doi: http://dx.doi.org/10.1590/S0080-62342000000300002

24. Barbosa DC, Mattos AT, Corrêa MH, Faria M, Ribeiro LC, Santos LL, Forstes AC. Visita domiciliar sob a percepção dos usuários da estratégia saúde da família. Medicina: Ribeirao Preto Online [internet] 2016 [acesso em 2017 jun]; 49(4): 360-366. doi: http://dx.doi.org/10.11606/ issn.2176-7262.v49i4p360-366

25. Santos EEP, Perin CB, Calza D, De Azevedo D, Oliveira SS, Amthauer C. Reflexões sobre visita domiciliar: estratégia para o cuidado qualificado e integral de indivíduos e famílias. Anuário Pesquisa e Extensão Unoesc São Miguel do Oeste, 2017; 2: e14084.

26. Thiago SCS, Tesser CD. Percepção de médicos e enfermeiros da Estratégia de Saúde da Família sobre terapias complementares. Rev. Saúde Pública. [internet] 2011 [acesso em 2017 jun 10];45 (2): 249-257. doi: http://dx. doi.org/10.1590/S0034-89102011005000002

27. Nóbrega AL, Ugulino PTD, Cajá DF, Dantas AEF. A importância da orientação dos profissionais das equipes de saúde da família acerca do uso da fitoterapia. Revista Brasileira de Educação e Saúde. 2017; 7(1): 43-48.

28. Egry EY, Fonseca R MGS, Oliveira MAC. Ciência, Saúde Coletiva e Enfermagem: destacando as categorias gênero e geração na episteme da práxis. Rev. bras. enferm. [internet] 2013 [acesso em 2017 jan 20]; 8(66):119-133. doi: http://dx.doi.org/10.1590/S0034-71672013000700016

29. Alves ED. Política de Educação e desenvolvimento para o SUS-Caminhos para a Educação Permanente em Saúde. Gestão e Saúde. [internet] 2017 [acesso em 2018 jan 20]; 1(1): 2- 3. doi: http://dx.doi.org/10.18673/gs.v1i1.24267

30. Moreira KS, Almeida LC, Vieira MA, Melo C. Educação permanente e qualificação profissional para atenção básica. Saúde e Pesquisa. [internet] 2017 [acesso em 2018 mai 23];10(1): 101-109. doi: dx.doi.org/10.17765/ 1983-1870.2017v10n1p101-109 